\section{PTH-011 ROLE OF THE NEW-GENERATION CHOLANGIOSCOPY IN THE DIAGNOSIS OF INDETERMINATE BILIARY LESIONS: A MULTI-CENTRE STUDY}

${ }^{1,2}$ Noor Bekkali*, ${ }^{3}$ Nick Church, ${ }^{4}$ Alison Winstanley, ${ }^{2}$ George Goodchild, ${ }^{2}$ Sham Direkze, ${ }^{1}$ Kofi Oppong, ${ }^{2}$ George Webster. ${ }^{1} H P B$ Unit, Freeman Hospital, Newcastle, UK; ${ }^{2}$ Pancreatobiliary unit, UCLH, London, UK; ${ }^{3} \mathrm{GI}$ department, Royal Infirmary, Edinburgh, UK; ${ }^{4}$ Department of Histopathology, UCLH, London, UK

\subsection{6/gutjnl-2018-BSGAbstracts.33}

Background Accurate diagnosis in patients with indeterminate biliary strictures remains challenging. Advances in cholangioscope design might facilitate improved visual and pathological diagnosis.

Objective To compare the diagnostic yield between brushings, cholangioscopy-directed Spybite biopsies (SB) and visual impression at cholangioscopy in patients presenting with indeterminate strictures referred for cholangioscopy.

Methods Prospective audit data was collected from 3 tertiary centres between June 2015-August 2017. Continuous data was expressed in mean values with 95\% confidence intervals (CI) and categorical data was analysed using chi-square test.

\begin{tabular}{|c|c|c|c|c|}
\hline & Brush $n=31$ & SB $n=58$ & $\begin{array}{l}\text { Visual } \\
\text { impression } \\
\mathrm{n}=70\end{array}$ & $\begin{array}{l}\text { Combined } \\
\text { SB+visual } \\
\text { impression }\end{array}$ \\
\hline \multirow[t]{2}{*}{ Sensitivity } & $33 \%(95 \% \mathrm{Cl}$ & $44 \%(95 \% \mathrm{Cl}$ & $73 \%(95 \% \mathrm{Cl}$ & $58 \%(95 \% \mathrm{Cl}$ \\
\hline & $12 \%-62 \%)$ & $23 \%-66 \%)$ & $50 \%-89 \%)$ & $42 \%-72 \%)$ \\
\hline \multirow[t]{2}{*}{ Specificity } & $100 \%(95 \% \mathrm{Cl}$ & $100 \%(95 \% \mathrm{Cl}$ & $67 \%(95 \% \mathrm{Cl}$ & $81 \%(95 \% \mathrm{Cl}$ \\
\hline & $79 \%-100 \%)$ & $90 \%-100 \%)$ & $51-80)$ & $71 \%-59 \%)$ \\
\hline \multirow[t]{2}{*}{ PPV } & $100 \%(95 \% \mathrm{Cl}$ & $100 \%(95 \% \mathrm{Cl}$ & $52 \%(95 \% \mathrm{Cl}$ & $63 \%(95 \% \mathrm{Cl}$ \\
\hline & NA) & $\mathrm{NA})$ & $40 \%-63 \%)$ & $51 \%-74 \%)$ \\
\hline \multirow[t]{2}{*}{ NPV } & $62 \%(95 \% \mathrm{Cl}$ & $73 \%(95 \% \mathrm{Cl}$ & $83 \%(95 \% \mathrm{Cl}$ & $77 \%(95 \% \mathrm{Cl}$ \\
\hline & $53 \%-70 \%)$ & $65 \%-79 \%)$ & $71 \%-91 \%)$ & $70 \%-83 \%)$ \\
\hline
\end{tabular}

PPV: positive predictive value; NPV: negative predictive value; SB: Spybite

Results 76 (41 M) patients, mean age 57 years (95\% CI 5362), had cholangioscopy for indeterminate strictures. Six patients were excluded from analysis as they were lost to follow-up $(n=3)$ or were awaiting further investigations $(n=3)$. Seven patients $(10 \%)$ had strictures in association with stones. Of 69 (90\%) with indeterminate strictures 40 (53\%) had had previous negative brush cytology, 9 (11.8\%) had had non-diagnostic FNA, and one had negative intraductal biopsies. Brushings were repeated at cholangioscopy in 31 patients, with sensitivity and specificity of 33\% (95\%CI 12\%-62\%) and $100 \%$ (95\%CI 79\%-100\%) for malignancy, respectively. Cholangioscopy guided SBs were taken in 58 patients and SB were not taken in 12 patients due to: stones mimicking strictures $n=7$, normal mucosa $n=3$, Mirrizi $n=1$, scope impassable due to stricture $\mathrm{n}=1$. The $\mathrm{SB}$ had sensitivity and specificity of $44 \% \quad$ (95\%CI 23\%-66\%) and 100\% (95\%CI 90\%-100\%), respectively. Overall, a mean number of 4.6 (95\% CI 3.75.5) SB samples were taken per patient with median size $2.2 \mathrm{~mm}$ (range 0.9-7). There was no difference in size between reported sufficient $1.5(0.5-2.4) \mathrm{mm}$ or insufficient $1.9 \mathrm{~mm}(1.5-2.3)$ biopsies. Pathologist reported insufficient (PANC-1) SB biopsies in 6 patients (10\%) and these correlated with more false negative (FN) findings compared to sufficient biopsies $(p=0.001)$. Cholangioscopic views were excellent in all patients and had sensitivity and specificity of $73 \%$ (95\% CI 50\%-89\%) and 67\% (95\%CI 51-80), respectively. Sensitivity and specificity for SB were significantly different between those for visual impression $(p=0.0004)$.

Conclusion The new generation cholangioscope has acceptable diagnostic yield, where visual impression exceeds the conventional SB confirmation Despite excellent visualisation of indeterminate strictures a definitive visual diagnosis remains challenging. It is hoped that further improvements in tissue acquisition technologies might advance pathological diagnosis.

\section{PTH-012 THE IMPACT OF CHOLANGIOSCOPY ON RADIATION EXPOSURE IN ERCP}

${ }^{1,2}$ Noor Bekkali ${ }^{*}{ }^{3}$ Anisha Bhagwanani, ${ }^{3}$ Manil Chouhan, ${ }^{2}$ MG Keane, ${ }^{2}$ George Webster. ${ }^{1}$ Freeman Hospital, Newcastle, UK; ${ }^{2} P B$-unit, UCLH, London, UK; ${ }^{3}$ Radiology department, UCLH, London, UK

\subsection{6/gutjnl-2018-BSGAbstracts.34}

Introduction Endoscopic retrograde cholangio-pancreatography (ERCP) remains the standard tool for biliary stone extraction, biliary drainage and intraductal stricture assessment. Cholangioscopy allows direct intraductal visualisation without requiring fluoroscopy. Therefore, the aim of this study was to assess radiation dose during ERCP in patients with stones or strictures with or without cholangioscopy.

Methods Data from patients referred for cholangioscopy between June 2015 and December 2016, for difficult biliary stones (following failed stone extraction with conventional ERCP), or to acquire tissue diagnosis for indeterminate strictures was prospectively collected. In all patients with stones, first conventional ERCP was attempted, using mechanical lithotripsy and or sphincteroplasty combined with balloon trawls before cholangioscopy was attempted.

Radiation exposure was indirectly measured using total dose area product (DAP) measured in Grey per square $\mathrm{cm}$ $\left(\mathrm{Gy} / \mathrm{cm}^{2}\right)$. Continuous variable differences were assessed using Student t-tests.

Results A total of 194 patients, mean age $62 \pm 18$ years [48\% male] were included based on intention to undergo cholangioscopy. Indications comprised: 128 with stone disease, 56 strictures, 7 stone and stricture and 3 'other'. Conventional ERCP for stone clearance sufficed for 29 (23\%) patients without cholangioscopy (group I); 1 patient had conventional ERCP for a stricture. Cholangioscopy was needed in 99 (77\%) patients (group II) for stones and 55 (98\%) patients referred for strictures (group III).

The mean DAP in group I was $85 \pm 661$ compared to 1912 $\pm 1817 \mathrm{~Gy} / \mathrm{cm}^{2}$ in group II; $p=0.003$. Radiation dose for conventional ERCP radiation for stones was comparable to the dose $\left(857 \pm 921 \mathrm{~Gy} / \mathrm{cm}^{2}\right)$ needed for stricture assessment for group III; $p=0.98$. The mean duration of the procedure (minutes) was $48 \pm 20$ in group I, vs $72 \pm 27$ in group II; $\mathrm{p}=0.0001$. For strictures necessitating cholangioscopy, 49 \pm 19 min were needed for assessment which was comparable to group $\mathrm{I} ; \mathrm{p}=0.92$. Radiation dosages per minute were persistently higher in group I compared to group II; $17 \pm 12$ vs $29 \pm 24 \mathrm{~Gy} / \mathrm{cm}^{2} / \mathrm{min}(\mathrm{p}=0.02)$. As only 1 patient had stricture not necessitating cholangioscopy, no comparison was made between group III for above findings.

Conclusion Cholangioscopy for stones adds considerably to procedure duration and total radiation dose compared to 
ERCP alone for stone disease. This likely reflects the high complexity of stone cases necessitating cholangioscopy. Of note, the radiation exposure/minute for ERCP with cholangioscopy in stone disease is no different to conventional ERCP. Our data on cholangioscopy in stricture assessment suggests that this may enhance diagnostic information (ie direct visualisation) without adding procedure time or radiation exposure.

\section{PTH-013 A VALIDATED COMFORT SCORE FOR GASTROSCOPY TOLERATION}

F Betteridge*, E Saunsbury, L Materacki, K Yong, D Murugiah, B Colleypriest. Royal United Hospital, Bath, UK

10.1136/gutjnl-2018-BSGAbstracts.35

Introduction Whilst comfort scoring is an auditable standard for quality in colonoscopy, there is currently no widely accepted comfort score for gastroscopy. We developed a five point gastroscopy toleration score (GTS) and validated correlation of scoring between endoscopists, patients and nursing staff.

Methods A GTS was developed via consensus on a gastroscopy list (figure 1). Trial 1 of the finalised 'Bath GTS' involved a double blind collection from the endoscopist and two nurses during 47 gastroscopies to determine inter-observer correlation between staff. Trial 2 involved a double blind collection from the endoscopist, nurse and unsedated patient during 50 additional cases. Krippendorff's alpha $(\alpha)$ coefficient was calculated to assess inter-rater reliability. We then analysed sub-group matches between endoscopist-nurse, endoscopistpatient and nurse-patient.

Results A total of 97 gastroscopies were included in the analysis. In Trial 1 the inter-rater correlation between nurses and endoscopist was excellent (Krippendorff's $\alpha=0.811$ (95\% CI $0.73-0.88)$ ). There were no matched scores in 1 case $(2.1 \%)$, at least two matched scores in 46 cases $(97.9 \%)$ and three matched scores in 34 cases (72.3\%).

\section{Bath Gastroscopy Toleration Score (GTS)}

0- Still throughout, may retch at intubation

1- Minimal retching $(<25 \%$ of procedure time)

2- Frequent retching ( $>25 \%$ of procedure time)

3- Attempts to handle and/or remove gastroscope

4- Extubates themselves or withdraws consent

\section{Abstract PTH-013 Figure 1}

Trial 2 demonstrated at least two matched scores in all 50 cases $(100 \%)$ and three matched scores in 39 cases $(78 \%)$ with excellent correlation between raters $(\alpha=0.833$ (95\% CI $0.75-0.90)$ ). Subgroup analysis demonstrated that of the gastroscopies with only two matched scores, endoscopist-nurse matches totalled 8 cases $(72.3 \%)$, endoscopist-patient matches totalled 2 cases (18.2\%) and nurse-patient matches 1 case
(9.10\%). Krippendorff's $\alpha$ for these subgroups were 0.930 (95\% CI $0.84-1.00), 0.800 \quad(95 \%$ CI $0.63-0.93)$ and 0.774 (95\%CI 0.60-0.92) respectively. Final analysis comparing endoscopist-nurse matched scores from all 97 gastroscopies showed significant agreement ( $\alpha=0.858$ (95\%CI $0.81-0.91)$ ).

Conclusions We have presented a simple yet effective GTS which was validated for inter-observer correlation between endoscopists and nurses with statistically significant agreement. Furthermore, there is excellent correlation when the GTS from unsedated patients is compared with that of staff $(\alpha=0.833$ (CI $0.75-0.90)$. Given the agreement between unsedated patients and staff regarding procedure toleration, this scoring system could be applied to sedated patients. We would suggest that the Bath GTS is adopted as a validated national auditable outcome for gastroscopy with further work in progress to determine the standard.

\section{PTH-014 POST-POLYPECTOMY SURVEILLANCE IN THE ENGLISH BOWEL CANCER SCREENING PROGRAMME: A PROSPECTIVE COHORT STUDY, PRELIMINARY RESULTS}

${ }^{1,2}$ Stewart Bonnington*, ${ }^{2}$ Linda Sharp, ${ }^{1,2}$ Matt Rutter. ${ }^{1}$ North Tees and Hartlepool NHS Trust, Stockton-on-Tees, UK; ${ }^{2}$ Newcastle University, Newcastle Upon Tyne, UK

\subsection{6/gutjnl-2018-BSGAbstracts.36}

Introduction The English Bowel Cancer Screening Programme (BCSP) offers individuals aged 60 to 74 years guaiac FOB testing $(\mathrm{gFOBt})$, with an invitation for colonoscopy if positive. Of more than 300000 individuals who have attended for colonoscopy, over 45000 attended post-polypectomy surveillance (PPS) after having intermediate (IR) or high risk (HR) adenomas detected and resected at screening. It is established that screening reduces mortality from colorectal cancer (CRC). However, robust evidence to support PPS is lacking.

Methods Details were extracted from the BSCP database for individuals who attended PPS from the start of the BCSP in 2006 until 3/1/2017. Data were analysed using Stata 14.

Results 67,435 PPS episodes were performed in 45151 individuals. $60 \%$ of individuals had attended only 1 PPS episode, $34 \%$ attended $2,5 \%$ attended 3 , and $0.4 \%$ attended 4 or 5 .

PPS episodes per year greatly increased over the study period. In 2008, 453 episodes were attended, rising to 13698 in 2016 (figure 1).

$70 \%$ of those attending PPS were male. $44 \%$ were aged 60 64 at the time of index screening, 43\% aged 65-69, and 10\% aged 70-74. The oldest age group had a higher proportion $(68 \%)$ of HR individuals than in the younger groups.

Screening risk category varied with gender. Overall, $40 \%$ of females and $51 \%$ of males were HR (figure 2).

Overall, the findings at first PPS demonstrate a high proportion of individuals with no further adenoma found (table 1).

Conclusions PPS accounts for an increasing proportion of endoscopy workload in the BCSP and more broadly in the UK and internationally. These results demonstrate a low proportion of CRC or IR or HR adenomas diagnosed at PPS in the BCSP.

Individuals with HR adenomas at screening more often had further adenomas detected at first PPS when compared to those with IR adenomas at screening. HR individuals also had a higher probability of IR or HR adenomas at first PPS. CRC diagnosis at first PPS was low in both groups $(\leq 0.5 \%)$.

Further evaluation of the BCSP database is ongoing in order to identify subgroups most likely to benefit from PPS. 